José Esteve Pardo

Catedrático de Derecho Administrativo

Universidad de Barcelona

\title{
Ciencia y Derecho ante los riesgos para la salud. Evaluación, decisión y gestión
}

SUMARIO: I. PLANTEAMIENTO. II. EL TRÁNSITO DE LOS PELIGROS NATURALES A LOS RIESGOS TECNOLÓGICOS. LOS NUEVOS RETOS PARA EL DERECHO. 1. EL PRINCIPIO DE RESPONSABILIDAD ANTE LOS RIESGOS COMO DEBIDOS A LA ACCIÓN HUMANA. 2. LA AUSENCIA DE REFERENCIAS JURÍDICAS MATERIALES EN LA DECISIÓN Y GESTIÓN SOBRE RIESGOS. EL DOMINIO DE LA CIENCIA. 3. LA NUEVA ORIENTACIÓN. EL DERECHO REFLEXIVO. III. EL DERECHO ANTE LOS RIESGOS. LA ADOPCIÓN DE DECISIONES. 1. CIENCIA Y DERECHO EN LA VALORACIÓN Y DECISIÓN SOBRE RIESGOS. 2. LAS DECISIONES SOBRE RIESGOS. El DERECHO ANTE LA INCERTEZA. 3. DECIDIR EN LA INCERTEZA. El PRINCIPIO DE PRECAUCIÓN. IV. CONTROL Y ACTUACIÓN SOBRE RIESGOS. DE LA POLICÍA ADMINISTRATIVA A LA GESTIÓN DE RIESGOS. 1. LOS CRITERIOS DIFERENCIALES. 2. El RECURSO A LA AUTORREGULACIÓN. V. RESPONSABILIDADES DERIVADAS DE LOS RIESGOS. UN APUNTE.

\section{PLANTEAMIENTO}

La historia de la humanidad ha sido en muy buena medida la lucha para obtener de la naturaleza la satisfacción de sus necesidades vitales y para dominar los peligros naturales. La técnica, el desarrollo industrial y tecnológico ha sido el medio para conseguir esos objetivos. Para ORTEGA Y GASSET, la técnica es justamente eso: todo lo que el ser humano ha desarrollado para controlar y dominar la naturale- 
za; técnica es, en definitiva, todo lo que se interpone entre el ser humano y la naturaleza ${ }^{1}$.

Las sociedades desarrolladas se llaman así en definitiva por el desarrollo tecnológico que las ha liberado de muchas necesidades y peligros naturales. Las necesidades alimentarias y los peligros de inanición se han superado mediante una industria y tecnología de la alimentación. Los peligros de infecciones y enfermedades han sido en buena medida superados por la industria farmacéutica. Las necesidades energéticas se han satisfecho mediante la tecnología de la energía. Incluso una realidad tan primaria y natural como la distancia física ha sido superada por la tecnología. Se habla hoy de la muerte de la distancia. Se dice que la moderna sociedad de la información ha matado a la distancia. Las armas utilizadas para ello han sido también armas tecnológicas: las tecnologías de la comunicación.

Lo que ocurre es que estas tecnologías no son inocuas: eliminan peligros naturales pero generan sus propios riesgos. Aquí se sitúa una distinción sin duda convencional pero rica en consecuencias: que convencionalmente establecemos entre peligro y riesgo, que tiene una gran trascendencia en nuestro tema. El peligro tiene su origen en la naturaleza, en sus fuerzas o en sus limitaciones. El riesgo, en cambio, tiene su origen en una tecnología y afecta, principalmente, a la naturaleza -a bienes tales como el medio ambiente y, por supuesto, al ser humano ${ }^{2}$.

Sólo muy recientemente se han eliminado -al menos en Europa, otra cosa es la trágica situación del tercer mundo- los peligros de inanición, de plagas o enfermedades infecciosas - con la notable y dramática excepción de una enfermedad como es el SIDA-, y los peligros derivados de falta de energía para las necesidades vitales. Pero la eliminación de estos peligros ha sido mediante unas tecnologías que han generado sus propios riesgos: los riesgos de la tecnología alimentaria, los riesgos de la industria farmacéutica, los riesgos de la industria de la energía. Incluso la superación de la dis-

1 Por expresarlo en sus propias palabras, para ORTEGA, la técnica es «la reforma que el hombre impone a la naturaleza en vista de la satisfacción de sus necesidades...éstas eran imposiciones de la naturaleza al hombre. El hombre responde imponiendo a su vez un cambio a la naturaleza. Es, pues, la técnica, la reacción enérgica contra la naturaleza o circunstancia, que lleva a crear entre éstas y el hombre una nueva naturaleza puesta sobre aquélla, una sobrenaturaleza...la técnica es la reforma de la naturaleza, de esa naturaleza que nos hace necesitados y menesterosos». J. ORTEGA Y GASSET, Meditaciones de la técnica y otros ensayos sobre ciencia y filosofía, Revista de Occidente/Alianza, Madrid, 1996, p. 28.

2 Como señala Ulrich BECK, «el desarrollo de la industria y la tecnología nos ha conducido hacia la sociedad del riesgo», los riesgos ambientales, riesgos para salud, los riesgos que amenazan al propio planeta, derivados del desarrollo industrial y tecnológico son riesgos con los cuales las modernas sociedades han de convivir. La sociedad del riesgo, Barcelona, 1998, p. 30. 
tancia no deja de plantear agudos y debatidos problemas sobre los riesgos para la salud de algunas de sus tecnologías, como son las que utilizan las ondas electromagnéticas.

Es así cómo en los últimos tiempos ha variado sustancialmente la evolución de la humanidad en su relación con la naturaleza y con la tecnología. Si durante miles de años estuvo primordialmente expuesta a peligros naturales es sólo muy recientemente que ha cambiado su posición. No estamos ya expuestos a peligros naturales que nos han preocupado durante miles de años sino ante los riesgos derivados de nuevas tecnologías, de nuevos organismos naturales derivados de la manipulación tecnológica.

Todo esto supone un cambio total de escenario, un cambio con unas referencias milenarias que han acuñado nuestra cultura. Podemos comprobarlo en el libro más difundido en nuestra cultura que contempla un panorama ya superado. En la Biblia, las amenazas que se ciernen sobre los seres humanos son siempre amenazas naturales, peligros naturales. El Faraón de Egipto le reveló a José sus sueños: vio el peligro natural de las vacas flacas por falta de alimentos naturales, pero no vio el riesgo de las vacas locas derivado de la manipulación de piensos y su tecnología; vio el peligro de malas cosechas pero no el riesgo de los alimentos mutados genéticamente; sufrieron los daños de plagas y pestes pero no los riesgos de los pesticidas.

Particularmente nuestros cuerpos, nuestra salud, están cada vez más distanciados de la naturaleza. El segmento tecnológico que se interpone entre nosotros y la naturaleza es cada vez más dilatado y más denso. No estamos ya expuestos a los peligros de una naturaleza que estamos modificando en sus componentes más íntimos hasta hacerla del todo artificial.

No estamos ya expuestos a los peligros de las pestes sino a los riesgos de nuestros pesticidas; más: a los riesgos de los organismos que hemos mutado genéticamente para hacerlos inmunes a nuestros pesticidas; a los riesgos de los mosquitos que se han alimentado de esos organismos mutados genéticamente y que por tanto también ellos, los mosquitos, resultan inmunes a esos pesticidas; para combatir esos mosquitos se están creando ahora nuevos pesticidas que pueden generar, a su vez, sus propios riesgos ${ }^{3}$. Riesgos sobre los que existe un conocimiento muy escaso, puesto que derivan de organismos hasta ahora inexistentes, resultado de una manipulación genética.

3 Sobre la biotecnología agraria, vid. el esclarecedor artículo de M. ALMODÓvAR IÑESTA, «Aspectos jurídicos de la biotecnología agroalimentaria», Revista Interdisciplinar de Gestión Ambiental, n. 28, abril 2001, p. 1 y ss. 


\section{EL TRÁNSITO DE LOS PELIGROS NATURALES A LOS RIESGOS TECNOLÓGICOS. LOS NUEVOS RETOS PARA EL DERECHO}

Como ya he podido exponer con detalle en otro lugar ${ }^{4}$, las relaciones entre la técnica y el Derecho son cada vez más intrincadas y sitúan al Derecho en la tesitura de resolver, básicamente, tres grandes cuestiones o problemas.

\section{EL PRINCIPIO DE RESPONSABILIDAD ANTE LOS RIESGOS COMO DEBIDOS A LA ACCIÓN HUMANA}

El primero de ellos está relacionado con el impulso del desarrollo tecnológico y sus límites. Desde esta perspectiva, es necesario advertir de que los riesgos están siempre precedidos de decisiones humanas. Detrás de cualquier tecnología están las decisiones adoptadas en su investigación, en su desarrollo y en su aplicación. Decisiones públicas y privadas. Positivas o negativas. Explícitas o implícitas. Pero siempre decisiones humanas de las que somos, o hemos de ser, responsables 5 . Eso convierte a los riesgos tecnológicos en objeto de atención por el Derecho. Esta perspectiva no supone desde luego la criminalización del progreso o la puesta del progreso bajo sospecha. En la actualidad y en ciertos sectores puede extenderse la difusa percepción de que el Derecho y los juristas, ciertas leyes y ciertos juristas, están en contra del progreso tecnológico. Las cosas son mucho más sencillas: el progreso tecnológico que ha tenido, desde luego, logros muy positivos- se debe siempre a decisiones humanas y, por tanto, puede ser objeto de atención por el Derecho. El Derecho debe reparar y pronunciarse sobre quién adopta esas decisiones, con qué legitimación, con qué responsabilidades en su caso o con qué apoyos y estímulos si ese desarrollo tecnológico se considera positivo. Nada de eso ocurre con los peligros de la naturaleza que se deben a la fuerza inescrutable del destino. El destino que impone las fuerzas externas de la naturaleza puede ser objeto de la literatura, de la filosofía, pero no del Derecho.

\footnotetext{
4 Técnica, riesgo y Derecho. (Ariel), Barcelona, 1999.

5 En el plano filosófico es de interés -además de haber ejercido una apreciable influencia- la obra de Hans JONAS, El principio de responsabilidad (Ensayo de una ética para la civilización tecnológica), Barcelona, 1995.
} 


\section{LA AUSENCIA DE REFERENCIAS JURÍDICAS MATERIALES EN LA} DECISIÓN Y GESTIÓN SOBRE RIESGOS. EL DOMINIO DE LA CIENCIA

El segundo gran problema que los riesgos plantean es que, paradójicamente, aunque son objeto de atención creciente por el Derecho, las principales referencias sobre los mismos no las ofrece el Derecho, sino la ciencia. Un medicamento, un producto alimentario, puede cumplir exquisitamente con todas las exigencias legales y con todas las autorizaciones de la Administración, puede que esté siendo utilizado desde hace un tiempo pero eso no impide que nuevos conocimientos científicos descubran la existencia de un riesgo para la salud por el consumo de ese medicamento o ese alimento. La correcta adecuación de la valoración científica y valoración política y jurídica de los riesgos es así una cuestión crucial.

\section{LA NUEVA ORIENTACIÓN. EL DERECHO REFLEXIVO}

El tercer gran reto que plantean los riesgos es que tienen su origen en lo que es el eje central de las modernas sociedades: la industria y la tecnología. Además, normalmente proceden de la industria y la tecnología que cumple con el Derecho, reconocida por el Derecho y, en muchos casos, incentivada por el Derecho. En materia de riesgos el Derecho debe actuar en cierto modo contra sí mismo, debe hacerse reflexivo ${ }^{6}$. Los riesgos no se localizan en actividades ilegales y clandestinas sino en instalaciones, productos o actividades con pleno reconocimiento legal, en el tejido industrial y económico en el que en muy buena medida se apoya la sociedad. Cualquier actuación que se adopte en atención a los riesgos, como pueda ser su mero reconocimiento y su prevención, tiene un impacto en ese tejido económico. En materia de riesgos siempre hay que optar: entre el riesgo para la salud o el riesgo para un sector industrial por la retirada de un producto y la mala imagen entre consumidores. El caso de las vacas locas que ponía en cuestión a todo el sector de la industria cárnica y la industria de los piensos resulta bien elocuente al respecto. Pero también en el propio tratamiento de los riesgos debe optarse entre ellos: los riesgos también se combaten con tecnología, una tecnología antirriesgo que pueden generar los suyos propios.

Pasemos ahora a considerar cómo estos retos que los riesgos plantean, particularmente los riesgos para la salud y el medio ambiente, son

${ }^{6}$ Sobre el Derecho reflexivo, vid., recientemente, mi libro Autorregulación. Génesis y efectos. (Aranzadi), Pamplona, 2002, en especial, pp. 21 y ss. Con una perspectiva más general, BECK/GIDDENS/LASH, Modernización reflexiva. Política, tradición y estética en el orden social moderno, (Alianza), Madrid, 1996. 
afrontados por el Derecho en los momentos en que más resueltamente ha de encararse con ellos. Esos momentos o fases son, básicamente, tres: decisión sobre la admisibilidad de los riesgos, control y gestión de riesgos, responsabilidades que en torno a los riesgos pudieran plantearse.

\section{EL DERECHO ANTE LOS RIESGOS. LA ADOPCIÓN DE DECISIONES}

En la decisión sobre riesgos se plantea con mayor agudeza la tensión entre el elemento científico y el elemento jurídico. Estos dos elementos están ya netamente diferenciados en las más recientes regulaciones de la Unión Europea con relación a los riesgos sobre la salud, muy especialmente en materia de seguridad alimentaria. Se establecen así como dos poderes, el científico y el jurídico, que se atribuyen a organismos diferentes y que deben actuar con independencia. Una nueva división de poderes. El poder científico que se atribuye a Agencias independientes de nueva creación y el poder político y regulador de los órganos políticos ya existentes ${ }^{7}$.

\section{CIENCIA Y DERECHO EN LA VALORACIÓN Y DECISIÓN SOBRE RIESGOS}

Son funciones de distinto contenido las que se encomiendan a estos dos poderes. El poder científico tiene funciones de información, dictamen y, en definitiva, de valoración de riesgos, pero no de decisión. La legitimación científica, por el conocimiento experto y especializado, no alcanza así poder decisorio que corresponde a las instancias públicas que tengan atribuidas - por determinación constitucional en último término- tales funciones.

Pero más relevante tal vez que las determinaciones constitucionales y las regulaciones europeas sobre las funciones de la ciencia y el Derecho con relación a los riesgos, es la diferencia natural y de objetivos de estos dos poderes. Si a la ciencia no le compete la adopción de decisiones, ello no es sólo por falta de legitimación sino, sobre todo, porque ella misma no pretende decidir. No podemos esperar decisiones de la ciencia. No es sólo que la ciencia es prudente por naturaleza, sino que sus investigaciones, sus informes, sus resultados -frecuentemente ex-

7 Esta división de funciones entre la ciencia y el Derecho, entre la comunidad científica y el sistema jurídico, en lo relativo a la valoración y gestión de riesgos, es resueltamente asumida por la normativa europea más reciente. Así, en la Directiva (EC), 178/2002 del Parlamento Europeo y del Consejo sobre Seguridad Alimentaria. 
presados en probabilidades, en términos relativos- están permanentemente abiertos a la discusión y la controversia. Existen controversias científicas no resueltas en torno a muchos de los riesgos para la salud que son hoy objeto de debate político y social, y sobre los cuales el Derecho, las instancias y órganos habilitados para ello, deben adoptar -y de hecho han adoptado-importantes decisiones.

Y es que el Derecho debe decidir. Y debe decidir, cada vez más, en situaciones de debate científico, de incerteza científica en definitiva. El Derecho debe decidir también en el momento en que se le requiere para ello, aunque se trate de una situación de incerteza científica que la ciencia considere que sólo se superará dentro de muchos años. La comunidad científica afirma así con cierta unanimidad que los efectos en la salud humana de ciertos alimentos procedentes de la biotecnología, de nuevos medicamentos o de los campos electromagnéticos sólo se conocerán con una mínima certeza dentro de veinte o treinta años. Pero mucho antes, ahora, el Derecho y quienes están llamados a definirlo y aplicarlo han de adoptar decisiones muy importantes sobre estos posibles riesgos. El tiempo corre y se mide de manera bien diferente para la ciencia y para el Derecho.

Esa facultad y esa obligación de decidir son la grandeza y la servidumbre del Derecho y de sus operadores. Los legisladores deciden estableciendo las normas sobre riesgos, sobre la industria alimentaria, farmacéutica, de atención médica. Las Administraciones deciden cuándo autorizan o deniegan la comercialización de un nuevo alimento o de un nuevo cultivo. Los tribunales han de decidir siempre, tanto en la revisión de las decisiones administrativas como en las cuestiones que se les puedan plantear en otros frentes como el de la responsabilidad civil.

\section{LAS DECISIONES SOBRE RIESGOS. EL DERECHO ANTE LA INCERTEZA}

En esas decisiones sobre riesgos tecnológicos las referencias que ofrecen las normas jurídicas son muy limitadas, muchas veces inexistentes. Frecuentemente se trata, como ya se ha dicho, de productos, actividades e instalaciones que cumplen con las exigencias de las normas y que cuentan con las necesarias autorizaciones. Pero la apreciación y valoración de riesgos no corresponde al Derecho, sino a la ciencia. Y la ciencia, con frecuencia, no ofrece seguridades y en tales situaciones de incerteza debe decidir el Derecho.

¿Cómo decide el Derecho en la incerteza? En realidad no se trata de un problema nuevo. El Derecho romano se enfrentó con asiduidad a situaciones de incerteza. Había incerteza sobre si una persona desa- 
parecida continuaba viva a efectos del Derecho de familia. Tampoco se sabía en caso de un naufragio en qué orden murieron los miembros de una familia a efectos del Derecho de sucesiones. El Derecho resolvía y resuelve esas situaciones de incerteza mediante presunciones: la norma impone su certeza jurídica sobre la incerteza de los hechos. En otros casos, en los que deben decidir los tribunales la norma no adopta una decisión clara pero decanta la decisión de los tribunales mediante la imposición de la carga de la prueba.

No es de extrañar que en los últimos años y ante las situaciones de incerteza científica que envuelven diversas supuestos en los que ha de pronunciarse el Derecho se haya optado por la fórmula de las presunciones o por el traslado de la carga de la prueba, especialmente en casos de responsabilidad civil. Así ha ocurrido en la reciente legislación de varios Estados europeos en materia de responsabilidad por daños ambientales y así es de esperar que reaccione el Derecho ante otros problemas planteados por la obligación de decidir sobre riesgos en un entorno de incerteza ${ }^{8}$.

También recientemente se asiste a otra reacción del Derecho ante la incerteza no resuelta por la comunidad científica. Se diseña entonces por el Derecho una fórmula que podríamos denominar de institucionalización de la apreciación y valoración científica. Se diseña en suma una institución -con la tendencia, tan actual como imprecisa, a denominarla agencia-cualificada por su conocimiento experto en una materia y a ella se atribuye de manera prácticamente exclusiva -con el propósito de evitar el debate de la comunidad científica en terreno abierto- la apreciación científica y la valoración de riesgos. Claros exponentes de esta reacción del Derecho en los espacios de incerteza científica, particularmente en los que pueden plantearse riesgos para la salud humana son, por ejemplo, la Autoridad Europea para la Seguridad Alimentaria o, en el ámbito nacional, la Agencia Española de Seguridad Alimentaria ${ }^{9}$. Los ejemplos podrían reiterarse en otros ámbitos, como el de los fármacos, pero es en cualquier caso significativa la confirmación de esta tendencia tanto en el plano comunitario como en el nacional.

\section{DECIDIR EN LA INCERTEZA. EL PRINCIPIO DE PRECAUCIÓN}

Pero posiblemente la novedad más destacada como criterio para decidir en situaciones de incerteza es la afirmación del llamado principio

${ }^{8}$ Sobre estos movimientos y reajustes del Derecho, replanteando muchas referencias y posiciones de partida en materia de responsabilidad en los casos, tan frecuentes, en los que la complejidad y la incerteza envuelven la determinación de elementos tan relevantes como la relación de causalidad, vid. el último capítulo de mi libro, Técnica, riesgo y Derecho, cit.

9 Creada la primera por el Reglamento del Parlamento y la Comisión Europea 178/2002, de 28 de enero, y la segunda por la Ley 11/2001, de 5 de junio. 
de precaución. Es un principio explícitamente reconocido por el Derecho primario de la Unión Europea para ser aplicado en casos de riesgos para el medio ambiente, posteriormente extendido sin torsión alguna por la jurisprudencia y la doctrina a los riesgos para la salud ${ }^{10}$.

Aunque se trata de un principio poco precisado en cuanto a su contenido, creo que su fundamental operatividad es precisamente esa: la de que faculta a los órganos públicos -Gobierno, Administraciones, Tribunales- para que, en situaciones de incerteza, puedan adoptar decisiones fundadas en el principio de precaución y que difícilmente podrían adoptar de otra manera.

Las circunstancias de los casos en los que puede operar este principio ya las conocemos: alimentos, cultivos, productos, instalaciones que cumplen con todos los requisitos legales, pero que pueden generar un riesgo para la salud o para el medio ambiente. Como no se tiene certeza absoluta sobre la existencia del riesgo, sobre su gravedad, difícilmente se podría adoptar una decisión preventiva, negativa, denegando la comercialización de un producto, su retirada del mercado o el cierre de una instalación en base a la legalidad vigente que se observa rigurosamente por la empresa o instalación de que se trate. El principio de precaución ofrece cobertura para adoptar este tipo de decisiones, todas ellas en principio con una orientación preventiva y un contenido negativo: deniegan autorizaciones, retiran productos, cierran instalaciones, etc.

El principio de precaución tiene dos límites fundamentales. Uno deriva de su contenido intrínseco: la decisión negativa sólo puede adoptarse si se dan situaciones de incerteza. Los tribunales no han entrado en valoraciones científicas sobre la incerteza pero sí que han reaccionado, sobre todo, cuando no se han seguido hasta el final procedimientos o estudios que pudieran despejar posibles dudas. Los tribunales en estos casos han adoptado decisiones negativas hasta que tales estudios e informes no se lleven hasta el final ${ }^{11}$. Los tribunales no entran en definitiva en el espacio de la ciencia pero sí exigen que en ese espacio se hayan apurado las vías y procedimientos para llegar al más preciso conocimiento. En cambio, las Administraciones a través de sus órganos técnicos sí que han sido más proclives a entrar en valoraciones de contenido científico.

10 En concreto, desde que el Tratado de la Comunidad Europea fue modificado por el Tratado de Maastricht, el principio de precaución se advierte en el artículo 174 al establecer que «la política de la Comunidad en el ámbito del medio ambiente...se basará en los principios de cautela y de acción preventiva».

11 Muy relevante y significativa resulta a este respecto la Sentencia del Tribunal de Justicia de las Comunidades Europeas, 21 de marzo de 2000, resolviendo una cuestión prejudicial planteada por el Consejo de Estado francés sobre la normativa europea sobre organismos mutados genéticamente, concretamente sobre la autorización de una modalidad de maíz transgénico. 
El otro límite del principio de precaución está relacionado con sus efectos. Como todas las cuestiones de riesgo, la opción preventiva en atención a los riesgos sobre la salud tiene siempre efectos sobre el sistema económico y su estructura tecnológica. Las decisiones adoptadas de acuerdo con el principio de precaución tienen siempre un efecto negativo para el sistema económico y el comercio de productos, una cuestión a la que obviamente es muy sensible la Unión Europea. Por ello, las recientes regulaciones del Derecho comunitario establecen que las medidas adoptadas en base al principio de precaución deben ser proporcionadas y contener las restricciones para el comercio que resulten estrictamente necesarias para alcanzar el elevado nivel de protección de la salud asumido por la Comunidad.

\section{CONTROL Y ACTUACIÓN SOBRE RIESGOS. DE LA POLICÍA ADMINISTRATIVA A LA GESTIÓN DE RIESGOS}

Si la primera tarea con relación a los riesgos es la decisión sobre su admisión o rechazo, la segunda función es la del control y seguimiento de esos riesgos. Son dos aspectos muy relacionados: con mucha frecuencia se admite un riesgo con la condición de que esté bajo un régimen de control riguroso.

En materia de control de riesgos se dan importantes novedades que afectan a conceptos y tradiciones del Derecho europeo. En este punto la gestión de riesgos tiende a desplazar las formas de actividad administrativa de intervención que, al menos en los países continentales, se habían articulado en torno al concepto de policía administrativa. La comparación del modelo de policía con el de gestión de riesgos nos da una idea de las novedades que la moderna sociedad de riesgo produce en el Derecho.

\section{LOS CRITERIOS DIFERENCIALES}

La policía administrativa, la policía sanitaria y de los alimentos, se concentra en la represión de actividades ilegales que pudieran causar daños a la salud (comercio y productos clandestinos, adulteración de alimentos, etc.) mientras que la gestión de riesgos actúa sobre actividades y productos perfectamente ajustados a las exigencias legales. En realidad, en la gestión de riesgos no se toman en consideración las referencias jurídicas de las normas, sino las referencias de la valoración de riesgos desde las instancias científicas. 
La policía administrativa aspira siempre a un estado de perfección: a un orden público, no sólo en lo relativo a la seguridad pública, sino también, sobre todo las llamadas policías especiales, a un orden público económico, sanitario, alimentario. La policía administrativa actúa cuando se producen perturbaciones de ese orden y su objetivo es, precisamente, restablecer ese orden que supone, al menos idealmente, una situación de perturbación cero. En cambio, la gestión de riesgos no se plantea el objetivo del riesgo cero porque sencillamente no existe. De lo que se trata es de optar entre riesgos. Por eso, el concepto final de la gestión de riesgos no es el orden público sino el de riesgo permitido. Un concepto con el que ya opera buena parte de la doctrina del Derecho penal.

Los medios de la policía administrativa son las medidas unilaterales administrativas, generalmente con carácter de autoridad: sanciones, autorizaciones, actos unilaterales de intervención. La gestión de riesgos, en la medida en que incide sobre actividades y productos en principio legales y que constituyen el eje del progreso ha de operar prudentemente, de manera reflexiva, y contando con la colaboración de la industria y la tecnología que genera estos riesgos. Una colaboración necesaria por la complejidad técnica de muchas cuestiones.

\section{EL RECURSO A LA AUTORREGULACIÓN}

Todo esto nos pone en contacto con la autorregulación y el destacado protagonismo que sin duda tendrá en todo lo relativo a la gestión de riesgos. Por una parte, la autorregulación viene impuesta en todos los sectores de riesgo; por hipótesis, el riesgo tiene siempre un destacado componente tecnológico y, con él, una importante carga de complejidad. Esa complejidad sólo es conocida y dominada, aunque no siempre, por los propios profesionales y expertos que operan en cada uno de estos sectores. Según la teoría de los sistemas se advertirían aquí, sólo en lo relativo a riesgos para la salud, diversos sistemas diferenciados, como puedan ser el de los alimentos, el de los productos farmacéuticos, el de la biotecnología; cada uno con sus propios profesionales y expertos, con sus propias normas técnicas, sus propios sistemas técnicos de control; en definitiva, con sus propias referencias, con su tendencia a configurarse en lo que la sociología de sistemas denomina sistemas autorreferenciales ${ }^{12}$.

Pero la autorregulación viene también impuesta por el Derecho. En las materias que contemplamos, no se trata, como a primera vista

12 Las cuestiones aquí apuntadas las desarrollo en mi libro Autorregulación. Génesis y efectos, op. cit. 
pudiera pensarse, de una orientación desreguladora sino, justamente, de todo lo contrario. El Derecho ambiental ofrece múltiples ejemplos en los que un aparente aumento de la colaboración privada en la toma de decisiones públicas esconde niveles de intervención administrativa históricamente desconocidos. Las remisiones a la utilización de la mejor tecnología disponible en la concesión de autorizaciones administrativas es una muestra de ello ${ }^{13}$.

También en el Derecho alimentario puede observarse esta tendencia. El Derecho europeo y los Derechos nacionales han extremado el control sobre todas las fases de producción de los alimentos (desde su origen hasta la mesa). Se establece así el concepto de trazabilidad y el sistema de control de puntos críticos en los procesos de producción de alimentos con un detalle y una especialización en cada uno de los alimentos que resultaría inabarcable para la Administración pública y sus agentes. Entran entonces en juego los mecanismos de autorregulación que suponen la atribución a los propios sectores productivos de sus propias normas, fijación de códigos de buenas prácticas, sistemas de acreditación ${ }^{14}$, de certificación, y de autocontrol.

Naturalmente esto plantea el problema de la atribución de la gestión y control de riesgos a quienes los generan. El problema de la captura del regulador público por la autorregulación privada. Se trata de un problema general, que rebasa el ámbito de nuestro tema, pero que reclama que por parte del poder público, por parte del legislador y la Administración pública, se defina con precisión el marco de la autorregulación privada y se establezca también una especie de control de puntos críticos en los procesos de autorregulación privada.

\section{RESPONSABILIDADES DERIVADAS DE LOS RIESGOS. UN APUNTE}

El tercer reto importante que el riesgo plantea es el relativo a la responsabilidad. De acuerdo con el concepto que utilizamos, detrás de los riesgos tecnológicos se encuentran siempre decisiones humanas. Decisiones de las que se ha de ser responsable y en las que, en caso de que se produzcan daños, se debe asumir la responsabilidad. Una responsabilidad que debe incorporar no sólo las consecuencias inmediatas de una acción o decisión, sino también sus consecuencias futuras.

El problema principal que se plantea en lo referente a la responsabilidad tiene su origen, una vez más, en la incerteza científica y de co-

13 Vid. J. Esteve PARDO, «La adaptación de las licencias a la mejor tecnología disponible», $R A P$, n. 149,1999 , p. 37 y ss. 
nocimiento que envuelve a los modernos riesgos tecnológicos para la salud. La incerteza vuelve a ser aquí un elemento fundamental. El supuesto característico del riesgo no es cuando se incumplen las normas jurídicas y se produce un daño. Eso no plantea problema alguno. El problema característico de la sociedad de riesgo se da, justamente, cuando, cumpliéndose las exigencias y referencias jurídicas, se produce un daño.

Las referencias jurídicas resultan insuficientes, al menos no ofrecen la certeza necesaria en sectores dominados por una tecnología cambiante cuyos efectos, en muchos casos, no se conocen con una mínima precisión hasta el paso de un tiempo. La propia industria farmacéutica reconoce que los efectos reales de un fármaco no se conocen con exactitud en sus periodos de pruebas sino cuando ya se comercializan y se administran a un número muy alto -cientos de miles-de pacientes o consumidores. En el caso de los productos transgénicos, o alimentos que incorporan nuevas tecnologías, la comunidad científica admite que no conoce todos sus posibles efectos. En general, tratándose de nuevas tecnologías, existe siempre un margen de desconocimiento. $\mathrm{Y}$ como la sucesión de nuevos productos y tecnologías es cada vez más rápida y constante, son, en principio, mayores las posibilidades de que se produzcan efectos negativos que no se conocían cuando se implantó, o incluso se autorizó, el producto o la tecnología.

Todo ello nos lleva a la debatida cuestión de la responsabilidad por los riesgos desconocidos o los riesgos de desarrollo. Una cuestión que fue directamente abordada pero no resuelta por la Directiva de 1985, relativa a la responsabilidad por productos defectuosos. No fue resuelta por no alcanzarse un acuerdo sobre este punto concreto. La Directiva deja la solución a los Estados miembros. En cualquier caso, una mayoría significativa de Estados europeos ha fijado el sector de los riesgos para la salud -más concretamente, el de alimentos y medicamentoscomo el sector en el que la responsabilidad corresponde al productor. En diversos informes de la Unión Europea sobre la aplicación de esa Directiva se señala también al sector de la salud como el más apropiado para imponer la responsabilidad sin que pueda alegarse la excepción del desconocimiento. En cualquier caso ya no cabe la excepción de fuerza mayor entendida como una fuerza externa, natural, allí donde siempre hay decisiones humanas.

14 Vid. M. TARRÉS VIVES, «Las nuevas referencias en el Derecho industrial: acreditación y normalización», Autonomías-Revista catalana de Derecho público, no 26, 2000, p. 140 y ss. 